\title{
Exact controllability for a one-dimensional wave equation with the fixed endpoint control
}

\author{
Lizhi Cui ${ }^{1}$, Yang Jiang ${ }^{2^{*}}$ and Yu Wang ${ }^{1}$
}

\section{"Correspondence:}

13478353900@163.com

${ }^{2}$ College of Math and Systematic

Science, Shenyang Normal

University, Shenyang, 110034, China

Full list of author information is

available at the end of the article

\begin{abstract}
This paper is devoted to the study of the exact controllability for a one-dimensional wave equation in domains with moving boundary. This equation characterizes the motion of a string with a fixed endpoint and the other a moving one. The control is put on the fixed endpoint. When the speed of the moving endpoint is less than the characteristic speed, by the Hilbert uniqueness method (HUM), exact controllability of this equation is established.
\end{abstract}

Keywords: exact controllability; non-cylindrical domain; wave equation

\section{Introduction}

Given $T>0$. Let us consider a non-cylindrical domain $\widehat{Q}_{T}^{k}$, defined by

$$
\widehat{Q}_{T}^{k}=\left\{(x, t) \in \mathbb{R}^{2} ; 0<x<\alpha_{k}(t) \text { for all } t \in(0, T)\right\},
$$

where

$$
\alpha_{k}(t)=1+k t, \quad 0<k<1
$$

Consider the following controlled wave equation in the non-cylindrical domain $\widehat{Q}_{T}^{k}$ :

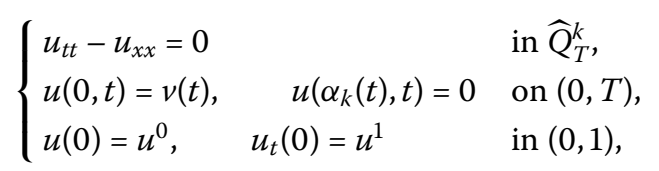

where $u$ is the state variable, $v$ is the control variable and $\left(u^{0}, u^{1}\right) \in L^{2}(0,1) \times H^{-1}(0,1)$ is any given initial value. Equation (1.2) may describe the motion of a string with a fixed endpoint and a moving one. The constant $k$ is called the speed of the moving endpoint. By [1], for $0<k<1$, any $\left(u^{0}, u^{1}\right) \in L^{2}(0,1) \times H^{-1}(0,1)$ and $v \in L^{2}(0, T),(1.2)$ admits a unique solution in the sense of transposition. The main purpose of this paper is to study the exact controllability of (1.2). In practical situations, many processes evolve in domains whose boundary has moving parts. A simple model is, e.g., the interface of an ice-water mixture when the temperature rises. To study the controllability problem of wave equations with

(c) 2015 Cui et al. This article is distributed under the terms of the Creative Commons Attribution 4.0 International License (http://creativecommons.org/licenses/by/4.0/), which permits unrestricted use, distribution, and reproduction in any medium, provided you give appropriate credit to the original author(s) and the source, provide a link to the Creative Commons license, and indicate if changes were made. 
moving boundary or free boundary is very significant. We first consider a one-dimensional wave equation with moving boundary when the moving endpoint moves along a line. In addition, for $0<k<1,(1.2)$ admits a unique solution in the sense of transposition. But for $k>1$ (1.2) admits a unique solution only when more boundary conditions are described. Now the controllability problem of this system is worth considering.

As is well known, there exists a variety of literature on the controllability and stabilization problems of wave equations in a cylindrical domain. However, there are only a few works of wave equations defined in non-cylindrical domains. We refer to [1-9] for some known results in this respect. Cavalcanti et al. [9] consider existence, uniqueness, and asymptotic behavior of global regular solutions of the mixed problem of the Kirchhoff nonlinear model for the hyperbolic-parabolic equation in non-cylindrical domains. The controllability problem of this system in [9] is open and is worth considering. In [2], the exact controllability of a multi-dimensional wave equation with constant coefficients in a non-cylindrical domain was established, while a control entered the system through the whole non-cylindrical domain. In [1-3], some controllability results for wave equations with Dirichlet boundary conditions in suitable non-cylindrical domains were investigated, respectively. But some additional conditions on the moving boundary were required, which render the method used in [1] and [3] inapplicable to the controllability problems of (1.2). In [1] and [3] in the one-dimensional case, the following condition seems necessary:

$$
\int_{0}^{\infty}\left|\alpha_{k}^{\prime}(t)\right| d t<\infty
$$

It is easy to check that this condition is not satisfied for the moving boundary in (1.2). In [7], instead of transforming the problem from a non-cylindrical domain into a cylindrical domain, Sun et al. studied the controllability problem directly in a non-cylindrical domain, when the control is put on the moving endpoint. But the method in [7] cannot be applied to the controllability problem in this paper. The control system of this paper is the same as that of [5] and [6]. But motivated by [2] and [7], we extend the result in [5] and [6], and the controllability result is obtained when $k \in(0,1)$. The key point is to define directly the energy function of a wave equation in the non-cylindrical domain and use the multiplier method to overcome these difficulties.

The rest of this paper is organized as follows. In Section 2, we give some preliminaries and main results. In Section 3, using the multiplier method, we give a growth estimate of the energy function and obtain two important inequalities used in Section 4. In Section 4, we prove that HUM works very well for (1.2).

\section{Preliminaries and main results}

The goal of this paper is to study the exact controllability of (1.2) in the following sense.

Definition 2.1 Equation (1.2) is called exactly controllable at the time $T$, if, for any initial value $\left(u^{0}, u^{1}\right) \in L^{2}(0,1) \times H^{-1}(0,1)$ and any target $\left(u_{d}^{0}, u_{d}^{1}\right) \in L^{2}\left(0, \alpha_{k}(T)\right) \times H^{-1}\left(0, \alpha_{k}(T)\right)$, one can always find a control $v \in L^{2}(0, T)$ such that the corresponding solution $u$ of (1.2) in the sense of a transposition satisfies

$$
u(T)=u_{d}^{0} \quad \text { and } \quad u_{t}(T)=u_{d}^{1}
$$


For $k \in(0,1)$, denote $T_{k}^{*}=\frac{e^{\frac{2 k(1+k)}{(1-k)^{3}}}-1}{k}$ for the controllability time. The main result of this paper is stated as follows.

Theorem 2.1 Suppose that $0<k<1$. For any given $T>T_{k}^{*}$, (1.2) is exactly controllable at time $T$ in the sense of Definition 2.1.

Remark 2.1 We can obtain the same result as that of this paper for a more general function $\alpha_{k}(t)$, as long as it meets the condition $0<\alpha_{k}^{\prime}(t)<1$.

Remark 2.2 In fact, if initial value $\left(u^{0}, u^{1}\right) \in H^{\frac{1}{2}}(0,1) \times H^{-\frac{1}{2}}(0,1)$, by extending $\widehat{Q}_{T}^{k}$ to a cylindrical domain, we can obtain the same controllability result when $\alpha_{k}(t) \in C[0 . T]$. The control is the trace on the fixed endpoint of the solution defined in the cylindrical domain. The extension method also applies to the moving endpoint control in non-cylindrical domain. But by the extension method, the controllability time is greater.

The key to the proof of Theorem 2.1 is in two important inequalities for the following homogeneous wave equation in the non-cylindrical domain:

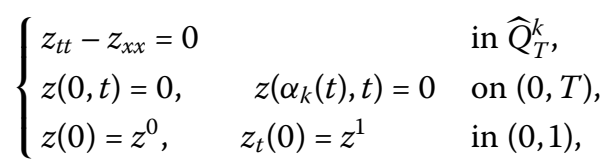

where $k \in(0,1),\left(z^{0}, z^{1}\right) \in H_{0}^{1}(0,1) \times L^{2}(0,1)$ is any given initial value. By [1], we see that (2.1) has a unique weak solution:

$$
z \in C\left([0, T] ; H_{0}^{1}\left(0, \alpha_{k}(t)\right)\right) \cap C^{1}\left([0, T] ; L^{2}\left(0, \alpha_{k}(t)\right)\right) .
$$

In the sequel, we denote by $C$ a positive constant depending only on $T$ and $k$, which may be different from one place to another.

We have the following two important inequalities. The proofs of the two important inequalities are given in Section 3.

Theorem 2.2 Let $T>T_{k}^{*}$. For any $\left(z^{0}, z^{1}\right) \in H_{0}^{1}(0,1) \times L^{2}(0,1)$, there exists a constant $C>0$ such that the corresponding solution $z$ of (2.1) satisfies

$$
C\left(\left|z^{0}\right|_{H_{0}^{1}(0,1)}^{2}+\left|z^{1}\right|_{L^{2}(0,1)}^{2}\right) \leq \int_{0}^{T} \alpha_{k}(t)\left|z_{x}(0, t)\right|^{2} d t \leq C\left(\left|z^{0}\right|_{H_{0}^{1}(0,1)}^{2}+\left|z^{1}\right|_{L^{2}(0,1)}^{2}\right) .
$$

\section{Observation: proof of Theorem 2.2}

In this section, in order to prove Theorem 2.2, we need the following lemmas.

Define the following weighted energy for (2.1):

$$
E(t)=\frac{1}{2} \int_{0}^{\alpha_{k}(t)}\left[\left|z_{t}(x, t)\right|^{2}+\left|z_{x}(x, t)\right|^{2}\right] d x \quad \text { for } t \geq 0
$$

where $z$ is the solution of (2.1). It follows that

$$
E_{0} \triangleq E(0)=\frac{1}{2} \int_{0}^{1}\left[\left|z^{1}(x)\right|^{2}+\left|z_{x}^{0}(x)\right|^{2}\right] d x
$$


Lemma 3.1 For any $\left(z^{0}, z^{1}\right) \in H_{0}^{1}(0,1) \times L^{2}(0,1)$ and $t \in[0, T]$, the corresponding solution $z$ of (2.1) satisfies

$$
E(t)-E_{0}=\frac{k\left(k^{2}-1\right)}{2} \int_{0}^{t}\left|z_{x}\left(\alpha_{k}(s), s\right)\right|^{2} d s .
$$

Proof Multiplying the first equation of (2.1) by $z_{t}$ and integrating on $\left(0, \alpha_{k}(s)\right) \times(0, t)$, for any $0<t \leq T$, we get

$$
0=\int_{0}^{t} \int_{0}^{\alpha_{k}(s)}\left[z_{t t}(x, s) z_{t}(x, s)-z_{x x}(x, s) z_{t}(x, s)\right] d x d s \triangleq I_{1}+I_{2}
$$

In the following, we calculate the above two integrals $I_{i}(i=1,2)$. It is easy to check that

$$
\begin{aligned}
I_{1} & =\int_{0}^{t} \int_{0}^{\alpha_{k}(t)} z_{t t}(x, s) z_{t}(x, s) d x d s \\
& =\int_{0}^{t}\left[\frac{\partial}{\partial s} \int_{0}^{\alpha_{k}(s)} \frac{1}{2}\left|z_{t}(x, s)\right|^{2} d x-\frac{1}{2} z_{t}^{2}\left(\alpha_{k}(s), s\right) \cdot k\right] d s \\
& =\int_{0}^{\alpha_{k}(t)} \frac{1}{2}\left|z_{t}(x, t)\right|^{2} d x-\int_{0}^{1} \frac{1}{2}\left|z^{1}\right|^{2} d x-\frac{1}{2} \int_{0}^{t} z_{t}^{2}\left(\alpha_{k}(s), s\right) \cdot k d s .
\end{aligned}
$$

By the definition of $\alpha_{k}$ and $z\left(\alpha_{k}(s), s\right)=0$, we have

$$
z_{x}\left(\alpha_{k}(s), s\right) \cdot k+z_{s}\left(\alpha_{k}(s), s\right)=0
$$

Therefore, it follows that

$$
I_{1}=\int_{0}^{\alpha_{k}(t)} \frac{1}{2}\left|z_{t}(x, t)\right|^{2} d x-\int_{0}^{1} \frac{1}{2}\left|z^{1}\right|^{2} d x-\frac{k^{3}}{2} \int_{0}^{t} z_{x}^{2}\left(\alpha_{k}(s), s\right) d s
$$

Further, by $z(0, s)=z\left(\alpha_{k}(s), s\right)=0$ on $(0, t)$, we have

$$
\begin{aligned}
I_{2}= & -\int_{0}^{t} \int_{0}^{\alpha_{k}(s)} z_{x x}(x, s) z_{t}(x, s) d x d s \\
= & -\int_{0}^{t} \int_{0}^{\alpha_{k}(s)}\left\{\frac{\partial}{\partial x}\left[z_{x}(x, s) z_{t}(x, s)\right]-z_{x}(x, s) z_{x t}(x, s)\right\} d x d s \\
= & -\int_{0}^{t} z_{x}\left(\alpha_{k}(s), s\right) z_{t}\left(\alpha_{k}(s), s\right) d s \\
& +\int_{0}^{t}\left[\frac{\partial}{\partial s} \int_{0}^{\alpha_{k}(s)} \frac{1}{2}\left|z_{x}(x, s)\right|^{2} d x-\frac{1}{2} z_{x}^{2}\left(\alpha_{k}(s), s\right) \cdot k\right] d s \\
= & k \int_{0}^{t} z_{x}^{2}\left(\alpha_{k}(s), s\right) d s+\int_{0}^{\alpha_{k}(t)} \frac{1}{2}\left|z_{x}(x, t)\right|^{2} d x \\
& -\int_{0}^{1} \frac{1}{2}\left|z_{x}^{0}\right|^{2} d x-\frac{k}{2} \int_{0}^{t} z_{x}^{2}\left(\alpha_{k}(s), s\right) d s \\
= & \int_{0}^{\alpha_{k}(t)} \frac{1}{2}\left|z_{x}(x, t)\right|^{2} d x-\int_{0}^{1} \frac{1}{2}\left|z_{x}^{0}\right|^{2} d x+\frac{k}{2} \int_{0}^{t} z_{x}^{2}\left(\alpha_{k}(s), s\right) d s .
\end{aligned}
$$


Therefore, by (3.3) and (3.4) and the definition of $E(t)$, we obtain

$$
E(t)-E_{0}=\frac{k\left(k^{2}-1\right)}{2} \int_{0}^{t}\left|z_{x}\left(\alpha_{k}(s), s\right)\right|^{2} d t
$$

Lemma 3.2 For any $\left(z^{0}, z^{1}\right) \in H_{0}^{1}(0,1) \times L^{2}(0,1)$ and $t \in[0, T]$, the corresponding solution $z$ of (2.1) holds,

$$
\left(1-k^{2}\right) \int_{0}^{t}\left|z_{x}\left(\alpha_{k}(s), s\right)\right|^{2} d s=2 t E(t)-2 \int_{0}^{1} x z^{1} z_{x}^{0} d x+2 \int_{0}^{\alpha_{k}(t)} x z_{t}(t) z_{x}(t) d x .
$$

Proof Multiplying the first equation of (2.1) by $2 x z_{x}$ and integrating on $\left(0, \alpha_{k}(s)\right) \times(0, t)$, for any $0<t \leq T$, from (3.2), we have

$$
\begin{aligned}
0= & \int_{0}^{t} \int_{0}^{\alpha_{k}(s)}\left[z_{t t} 2 x z_{x}-z_{x x} 2 x z_{x}\right] d x d s \\
= & \int_{0}^{t} \int_{0}^{\alpha_{k}(s)}\left\{\frac{\partial}{\partial t}\left(2 x z_{x} z_{t}\right)-\frac{\partial}{\partial x}\left(x z_{t}^{2}+x z_{x}^{2}\right)+z_{t}^{2}+z_{x}^{2}\right\} d x d s \\
= & \int_{0}^{t}\left[\frac{\partial}{\partial s} \int_{0}^{\alpha_{k}(s)} 2 x z_{x} z_{t} d x-2 \alpha_{k}(s) z_{x}\left(\alpha_{k}(s), s\right) z_{t}\left(\alpha_{k}(s), s\right) \cdot k\right] d s \\
& +\int_{0}^{t}\left[\alpha_{k}(s) z_{t}^{2}\left(\alpha_{k}(s), s\right)+\alpha_{k}(s) z_{x}^{2}\left(\alpha_{k}(s), s\right)\right] d s+2 \int_{0}^{t} E(s) d s \\
= & \int_{0}^{\alpha_{k}(t)} 2 x z_{x}(x, t) z_{t}(x, t) d x-\int_{0}^{1} 2 x z_{x}^{0} z^{1} d x \\
& -\int_{0}^{t} 2 \alpha_{k}(s) z_{x}\left(\alpha_{k}(s), s\right) z_{t}\left(\alpha_{k}(s), s\right) \cdot k d s \\
& -\int_{0}^{t}\left[\alpha_{k}(s) z_{t}^{2}\left(\alpha_{k}(s), s\right)+\alpha_{k}(s) z_{x}^{2}\left(\alpha_{k}(s), s\right)\right] d s+2 \int_{0}^{t} E(s) d s \\
= & \int_{0}^{\alpha_{k}(t)} 2 x z_{x}(x, t) z_{t}(x, t) d x-\int_{0}^{1} 2 x z_{x}^{0} z^{1} d x+2 \int_{0}^{t} E(s) d s \\
& +\left(k^{2}-1\right) \int_{0}^{t} \alpha_{k}(s) z_{x}^{2}\left(\alpha_{k}(s), s\right) d s .
\end{aligned}
$$

From this one concludes that

$$
\begin{aligned}
& \left(1-k^{2}\right) \int_{0}^{t} \alpha_{k}(s) z_{x}^{2}\left(\alpha_{k}(s), s\right) d s \\
& \quad=\int_{0}^{\alpha_{k}(t)} 2 x z_{x}(x, t) z_{t}(x, t) d x-\int_{0}^{1} 2 x z_{x}^{0} z^{1} d x+2 \int_{0}^{t} E(s) d s .
\end{aligned}
$$

Similarly, multiplying the first equation of (2.1) by $2 t z_{t}$ and integrating on $\left(0, \alpha_{k}(s)\right) \times$ $(0, t)$, for any $0<t \leq T$, we obtain

$$
\begin{aligned}
0 & =\int_{0}^{t} \int_{0}^{\alpha_{k}(s)}\left[z_{t t} 2 t z_{t}-z_{x x} 2 t z_{t}\right] d x d s \\
& =\int_{0}^{t} \int_{0}^{\alpha_{k}(s)}\left\{\frac{\partial}{\partial x}\left(2 t z_{x} z_{t}\right)-\frac{\partial}{\partial t}\left(t z_{t}^{2}+t z_{x}^{2}\right)+z_{t}^{2}+z_{x}^{2}\right\} d x d s .
\end{aligned}
$$


From this we get

$$
\left(1-k^{2}\right) \int_{0}^{t} k s z_{x}^{2}\left(\alpha_{k}(s), s\right) d s=2 \int_{0}^{t} E(s) d s-2 t E(t) .
$$

Therefore, (3.5) follows by (3.6) and (3.7).

By the above two lemmas, we obtain the following lemma concerning a growth estimate of the energy function.

Lemma 3.3 For any $\left(z^{0}, z^{1}\right) \in H_{0}^{1}(0,1) \times L^{2}(0,1)$ and $t \in[0, T]$, from the corresponding solution $z$ of (2.1) follows

$$
\frac{1-k}{(1+k) \alpha_{k}(t)} E_{0} \leq E(t) \leq \frac{1+k}{(1-k) \alpha_{k}(t)} E_{0}
$$

Proof From (3.1) and (3.5), it is easy to check that

$$
\frac{2}{k}\left(E(t)-E_{0}\right)=2 t E(t)+\int_{0}^{\alpha_{k}(t)} 2 x z_{x}(x, t) z_{t}(x, t) d x-\int_{0}^{1} 2 x z_{x}^{0} z^{1} d x
$$

By this equality, we get

$$
E_{0}-\int_{0}^{1} k x z_{x}^{0} z^{1} d x=\alpha_{k}(t) E(t)+\int_{0}^{\alpha_{k}(t)} k x z_{x}(x, t) z_{t}(x, t) d x
$$

On the other hand, for each $t \in(0, T)$ and $k \in(0,1)$, we obtain the estimate

$$
\left|\int_{0}^{\alpha_{k}(t)} x z_{x}(x, t) z_{t}(x, t) d x\right| \leq \alpha_{k}(t) E(t)
$$

From (3.9) and (3.10), we derive that

$$
\begin{aligned}
& (1-k) E_{0} \leq(1+k) \alpha_{k}(t) E(t), \\
& (1-k) E_{0} \geq(1-k) \alpha_{k}(t) E(t) .
\end{aligned}
$$

By this (3.8) follows.

Remark 3.1 Lemma 3.3 implies that

$$
\frac{1-k}{(1+k) \alpha_{k}(T)} E_{0} \leq E(T) \leq \frac{1+k}{(1-k) \alpha_{k}(T)} E_{0}
$$

In the following, we give the proof of Theorem 2.2.

Proof of Theorem 2.2 Step 1. Multiplying the first equation of (2.1) by $\left[x-\alpha_{k}(t)\right] z_{x}$ and integrating on $\left(0, \alpha_{k}(t)\right) \times(0, T)$, it follows that

$$
0=\int_{0}^{T} \int_{0}^{\alpha_{k}(t)}\left\{z_{t t}\left[x-\alpha_{k}(t)\right] z_{x}-z_{x x}\left[x-\alpha_{k}(t)\right] z_{x}\right\} d x d t \triangleq J_{1}+J_{2}
$$


Gui et al. Boundary Value Problems ( 2015) 2015:208

Page 7 of 10

In the following, we calculate the above two integrals $J_{i}(i=1,2)$. By $z(0, t)=0$ on $(0, T)$, it is easy to check that

$$
\begin{aligned}
J_{1}= & \int_{0}^{T} \int_{0}^{\alpha_{k}(t)} z_{t t}\left[x-\alpha_{k}(t)\right] z_{x} d x d t \\
= & \int_{0}^{T} \int_{0}^{\alpha_{k}(t)}\left\{\frac{\partial}{\partial t}\left[\left(x-\alpha_{k}(t)\right) z_{x} z_{t}\right]-k z_{x} z_{t}-\left(x-\alpha_{k}(t)\right) z_{x t} z_{t}\right\} d x d t \\
= & \left.\int_{0}^{\alpha_{k}(t)}\left[\left(x-\alpha_{k}(t)\right) z_{x} z_{t}\right] d x\right|_{0} ^{T}-k \int_{0}^{T} \int_{0}^{\alpha_{k}(t)} z_{x} z_{t} d x d t \\
& -\int_{0}^{T} \int_{0}^{\alpha_{k}(t)}\left(x-\alpha_{k}(t)\right) \frac{\partial}{\partial x}\left[\frac{1}{2} z_{t}^{2}\right] d x d t \\
= & \left.\int_{0}^{\alpha_{k}(t)}\left[\left(x-\alpha_{k}(t)\right) z_{x} z_{t}\right] d x\right|_{0} ^{T}-k \int_{0}^{T} \int_{0}^{\alpha_{k}(t)} z_{x} z_{t} d x d t \\
& -\left.\int_{0}^{T}\left(x-\alpha_{k}(t)\right) \frac{1}{2} z_{t}^{2}\right|_{0} ^{\alpha_{k}(t)} d t+\int_{0}^{T} \int_{0}^{\alpha_{k}(t)} \frac{1}{2} z_{t}^{2} d x d t \\
= & \left.\int_{0}^{\alpha_{k}(t)}\left[\left(x-\alpha_{k}(t)\right) z_{x} z_{t}\right] d x\right|_{0} ^{T}-k \int_{0}^{T} \int_{0}^{\alpha_{k}(t)} z_{x} z_{t} d x d t \\
& +\int_{0}^{T} \int_{0}^{\alpha_{k}(t)} \frac{1}{2} z_{t}^{2} d x d t .
\end{aligned}
$$

Further, it follows that

$$
\begin{aligned}
J_{2} & =-\int_{0}^{T} \int_{0}^{\alpha_{k}(t)} z_{x x}\left[x-\alpha_{k}(t)\right] z_{x} d x d t \\
& =-\int_{0}^{T} \int_{0}^{\alpha_{k}(t)}\left\{\left(x-\alpha_{k}(t)\right) \frac{\partial}{\partial x}\left[\frac{1}{2} z_{x}^{2}\right]\right\} d x d t \\
& =-\left.\int_{0}^{T}\left(x-\alpha_{k}(t)\right) \frac{1}{2} z_{x}^{2}\right|_{0} ^{\alpha_{k}(t)} d t+\int_{0}^{T} \int_{0}^{\alpha_{k}(t)} \frac{1}{2} z_{x}^{2} d x d t \\
& =-\int_{0}^{T} \alpha_{k}(t) \frac{1}{2} z_{x}^{2}(0, t) d x+\int_{0}^{T} \int_{0}^{\alpha_{k}(t)} \frac{1}{2} z_{x}^{2} d x d t .
\end{aligned}
$$

Therefore, by (3.12) and (3.13), we obtain

$$
\begin{aligned}
& \frac{1}{2} \int_{0}^{T} \alpha_{k}(t) z_{x}^{2}(0, t) d x \\
& \quad=\int_{0}^{T} E(t) d t-k \int_{0}^{T} \int_{0}^{\alpha_{k}(t)} z_{x} z_{t} d x d t+\left.\int_{0}^{\alpha_{k}(t)}\left[\left(x-\alpha_{k}(t)\right) z_{x} z_{t}\right] d x\right|_{0} ^{T} .
\end{aligned}
$$

Next, we estimate every term in the right side of (3.14).

For each $t \in[0, T]$, we have

$$
\begin{aligned}
& \left|\int_{0}^{\alpha_{k}(t)}\left[\left(x-\alpha_{k}(t)\right) z_{x} z_{t}\right] d x\right| \\
& \leq \int_{0}^{\alpha_{k}(t)}\left(\alpha_{k}(t)-x\right) \frac{1}{2}\left(z_{x}^{2}+z_{t}^{2}\right) d x \\
& \leq \alpha_{k}(t) E(t) .
\end{aligned}
$$


This inequality implies that

$$
\left|\int_{0}^{\alpha_{k}(t)}\left[\left(x-\alpha_{k}(t)\right) z_{x} z_{t}\right] d x\right|_{0}^{T} \mid \leq \alpha_{k}(T) E(T)+E_{0}
$$

Further,

$$
\left|k \int_{0}^{T} \int_{0}^{\alpha_{k}(t)} z_{x} z_{t} d x d t\right| \leq k \int_{0}^{T} E(t) d t
$$

Step 2. In the following, we give the proof of the first inequality in (2.2).

From (3.8), (3.11), and (3.14)-(3.16), it follows that

$$
\begin{aligned}
& \frac{1}{2} \int_{0}^{T} \alpha_{k}(t) z_{x}^{2}(0, t) d x \\
& \quad \geq(1-k) \int_{0}^{T} E(t) d t-\alpha_{k}(T) E(T)-E_{0} \\
& \quad \geq(1-k) \int_{0}^{T} \frac{1-k}{(1+k) \alpha_{k}(t)} E_{0} d t-\frac{1+k}{1-k} E_{0}-E_{0} \\
& \quad=\left[\frac{(1-k)^{2}}{(1+k) k} \ln (1+k T)-\frac{1+k}{1-k}-1\right] E_{0} .
\end{aligned}
$$

If $T>T_{k}^{*}$, we have $\frac{(1-k)^{2}}{(1+k) k} \ln (1+k T)-\frac{1+k}{1-k}-1>0$. Also,

$$
\int_{0}^{T} \alpha_{k}(t) z_{x}^{2}(0, t) d x \geq C\left[\frac{(1-k)^{2}}{(1+k) k} \ln (1+k T)-\frac{1+k}{1-k}-1\right]\left(\left|z^{0}\right|_{H_{0}^{1}(0,1)}^{2}+\left|z^{1}\right|_{L^{2}(0,1)}^{2}\right)
$$

Step 3. In the following, we prove the second inequality in (2.2).

From (3.8), (3.11), and (3.14)-(3.16), one concludes that

$$
\begin{aligned}
\frac{1}{2} & \int_{0}^{T} \alpha_{k}(t) z_{x}^{2}(0, t) d x \\
& \leq(1+k) \int_{0}^{T} E(t) d t+\alpha_{k}(T) E(T)+E_{0} \\
& \leq(1+k) \int_{0}^{T} \frac{1+k}{(1-k) \alpha_{k}(t)} E_{0} d t+\frac{1+k}{1-k} E_{0}+E_{0} \\
& =\left[\frac{(1+k)^{2}}{(1-k) k} \ln (1+k T)+\frac{1+k}{1-k}+1\right] E_{0} \\
& \leq C\left[\frac{(1+k)^{2}}{(1-k) k} \ln (1+k T)+\frac{1+k}{1-k}+1\right]\left(\left|z^{0}\right|_{H_{0}^{1}(0,1)}^{2}+\left|z^{1}\right|_{L^{2}(0,1)}^{2}\right) .
\end{aligned}
$$

Remark 3.2 Theorem 2.2 implies that, for any $\left(z_{0}, z_{1}\right) \in H_{0}^{1}(0,1) \times L^{2}(0,1)$, the corresponding solution $z$ of $(2.1)$ satisfies $z_{x}(0, \cdot) \in L^{2}(0, T)$.

Remark 3.3 It is easy to check that

$$
T_{0}^{*} \triangleq \lim _{k \rightarrow 0} T_{k}^{*}=\lim _{k \rightarrow 0} \frac{e^{\frac{2 k(1+k)}{(1-k)^{3}}}-1}{k}=\lim _{k \rightarrow 0} \frac{\frac{2 k(1+k)}{(1-k)^{3}}}{k}=2
$$


It is well known that the wave equation (1.2) in the cylindrical domain is null controllable at any time $T>T_{0}^{*}$.

Remark 3.4 By a similar method, we obtain the following.

Let $T>\frac{e^{\frac{2 k(1+k)}{(1-k)^{2}}}-1}{k}$. For any $\left(z^{0}, z^{1}\right) \in H_{0}^{1}(0,1) \times L^{2}(0,1)$, there exists a constant $C>0$ such that the corresponding solution $z$ of (2.1) satisfies

$$
C\left(\left|z^{0}\right|_{H_{0}^{1}(0,1)}^{2}+\left|z^{1}\right|_{L^{2}(0,1)}^{2}\right) \leq \int_{0}^{T} \alpha_{k}(t)\left|z_{x}\left(\alpha_{k}(t), t\right)\right|^{2} d t \leq C\left(\left|z^{0}\right|_{H_{0}^{1}(0,1)}^{2}+\left|z^{1}\right|_{L^{2}(0,1)}^{2}\right) .
$$

Therefore, we get the controllability of (1.2) when the control is put on the moving endpoint. But the controllability time is larger than that of [7].

\section{Controllability: proof of Theorem 2.1}

In this section, we prove the exact controllability for the wave equation (1.2) (Theorem 2.1) by the Hilbert uniqueness method.

Proof of Theorem 2.1 We divide the proof of Theorem 2.1 into two parts.

Step 1. First, we define a linear operator $\Lambda: H_{0}^{1}(0,1) \times L^{2}(0,1) \rightarrow H^{-1}(0,1) \times L^{2}(0,1)$.

For any $\left(z^{0}, z^{1}\right) \in H_{0}^{1}(0,1) \times L^{2}(0,1)$, denote by $z$ the corresponding solution of (2.1). Consider the following wave equation:

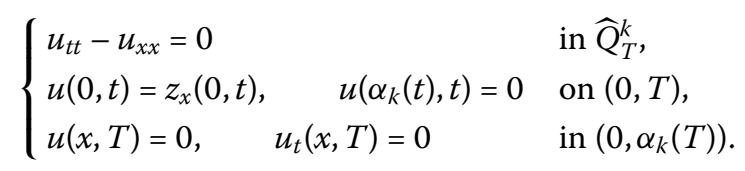

Then it is well known that (4.1) admits a unique solution in the sense of a transposition,

$$
u \in C\left([0, T] ; L^{2}\left(0, \alpha_{k}(t)\right)\right) \cap C^{1}\left([0, T] ; H^{-1}\left(0, \alpha_{k}(t)\right)\right) .
$$

Moreover, by Theorem 2.2 in Section 2, there exists a constant $C$ such that

$$
\begin{aligned}
& |u|_{C\left([0, T] ; L^{2}\left(0, \alpha_{k}(t)\right)\right) \cap C^{1}\left([0, T] ; H^{-1}\left(0, \alpha_{k}(t)\right)\right)} \\
& \quad \leq C\left|z_{x}(0, \cdot)\right|_{L^{2}(0, T)} \leq C\left(\left|z^{0}\right|_{H_{0}^{1}(0,1)}+\left|z^{1}\right|_{L^{2}(0,1)}\right) .
\end{aligned}
$$

Define a linear operator $\Lambda$ :

$$
\begin{aligned}
\Lambda: H_{0}^{1}(0,1) \times L^{2}(0,1) \rightarrow H^{-1}(0,1) \times L^{2}(0,1), \\
\quad\left(z^{0}, z^{1}\right) \mapsto\left(-u_{t}(x, 0), u(x, 0)\right),
\end{aligned}
$$

where we use $z$ to denote the solution of (2.1) associated to $z^{0}$ and $z^{1}$, and $u$ denotes the solution of (4.1) associated to $z$.

Step 2. That $\Lambda$ is an isomorphism is equivalent to the exact controllability of (1.2).

In fact, multiplying the first equation of (4.1) by $z$ and integrating on $\widehat{Q}_{T}^{k}$, we obtain

$$
\int_{0}^{1}\left(z^{1} u(x, 0)-z^{0} u_{t}(x, 0)\right) d x=\int_{0}^{T} z_{x}^{2}(0, t) d t
$$


Write $F=H_{0}^{1}(0,1) \times L^{2}(0,1)$ and denote by $F^{\prime}$ its conjugate space. Hence, we get

$$
\left\langle\Lambda\left(z^{0}, z^{1}\right),\left(u^{0}, u^{1}\right)\right\rangle_{F^{\prime}, F}=\int_{0}^{T} z_{x}^{2}(0, t) d t
$$

By Theorem 2.2, we see that $\Lambda$ is bounded and coercive. Therefore, by the Lax-Milgram theorem, it follows that $\Lambda$ is an isomorphism.

Since $\Lambda$ is an isomorphism, for any $\left(u^{0}, u^{1}\right) \in L^{2}(0,1) \times H^{-1}(0,1)$, there exists $\left(z^{0}, z^{1}\right) \in$ $H_{0}^{1}(0,1) \times L^{2}(0,1)$ such that

$$
\Lambda\left(z^{0}, z^{1}\right)=\left(-u^{1}, u^{0}\right)
$$

By the uniqueness of (4.1), $u$ is the solution of (1.2) associated to $v=z_{x}(0, \cdot)$. Furthermore, $\left(u(0), u_{t}(0)\right)=\left(u^{0}, u^{1}\right)$ and $\left(u(T), u_{t}(T)\right)=(0,0)$. Therefore, we get the exact controllability of (1.2).

\section{Competing interests}

The authors declare that they have no competing interests.

\section{Authors' contributions}

All results belong to LC, YJ, and YW. All authors read and approved the final manuscript.

\section{Author details}

${ }^{1}$ College of Applied Mathematics, Jilin University of Finance and Economics, Changchun, 130117, China. ${ }^{2}$ College of Math and Systematic Science, Shenyang Normal University, Shenyang, 110034, China.

\section{Acknowledgements}

This work is supported by the National Science Foundation of China 11171060, 11371084 and 11426157. Moreover, the authors are grateful to the anonymous referees for their constructive comments and suggestions, which led to an improvement of the original manuscript.

Received: 13 July 2015 Accepted: 4 November 2015 Published online: 14 November 2015

\section{References}

1. Milla Miranda, M: Exact controllability for the wave equation in domains with variable boundary. Rev. Mat. Univ. Complut. Madr. 9, 435-457 (1996)

2. Bardos, C, Chen, G: Control and stabilization for the wave equation, part III: domain with moving boundary. SIAM J. Control Optim. 19, 123-138 (1981)

3. Araruna, FD, Antunes, GO, Medeiros, LA: Exact controllability for the semilinear string equation in the non cylindrical domains. Control Cybern. 33, 237-257 (2004)

4. Cui, L, Liu, X, Gao, H: Exact controllability for a one-dimensional wave equation in non-cylindrical domains. J. Math. Anal. Appl. 402, 612-625 (2013)

5. Cui, L, Song, L: Exact controllability for a wave equation with fixed boundary control. Bound. Value Probl. 2014, 47 (2014)

6. Cui, L, Song, L: Controllability for a wave equation with moving boundary. J. Appl. Math. 2014, Article ID 827698 (2014)

7. Sun, H, Li, H, Lu, L: Exact controllability for a string equation in domains with moving boundary in one dimension. Electron. J. Differ. Equ. 2015, 98 (2015)

8. Benabidallah, R, Ferreira, J: On a hyperbolic-parabolic equations with nonlinearity of Kirchhoff-Carrier type in domains with moving boundary. Nonlinear Anal., Theory Methods Appl. 37, 269-287 (1999)

9. Cavalcanti, MM, Domingos Cavalcanti, VN, Ferreira, J, Benabidallah, R: On global solvability and asymptotic behaviour of a mixed problem for a nonlinear degenerate Kirchhoff model in moving domains. Bull. Belg. Math. Soc. Simon Stevin 10, 179-196 (2003) 